\title{
A Genome-Wide Pharmacogenetic Study of Growth Hormone Responsiveness
}

\author{
Andrew Dauber, ${ }^{1}$ Yan Meng, ${ }^{2}$ Laura Audi, ${ }^{3}$ Sailaja Vedantam, ${ }^{2}$ \\ Benjamin Weaver, ${ }^{2}$ Antonio Carrascosa, ${ }^{3}$ Kerstin Albertsson-Wikland, ${ }^{4}$ \\ Michael B. Ranke, ${ }^{5}$ Alexander A. L. Jorge, ${ }^{6}$ Jose Cara, ${ }^{7}$ Michael P. Wajnrajch, ${ }^{7}$ \\ Anders Lindberg, ${ }^{8}$ Cecilia Camacho-Hübner, ${ }^{7}$ and Joel N. Hirschhorn ${ }^{2}$ \\ ${ }^{1}$ Division of Endocrinology, Children's National Hospital, Washington, DC 20010; 'Division of \\ Endocrinology, Boston Children's Hospital, and Program in Medical and Population Genetics, Broad \\ Institute, Harvard Medical School, Boston, Massachusetts 02115; ${ }^{3}$ Department of Pediatrics, Institut \\ de Recerca (VHIR), Hospital Vall d'Hebron, Centre for Biomedical Research on Rare Diseases (CIBERER), \\ Autonomous University, Barcelona, Spain; ${ }^{4}$ Department of Physiology/Endocrinology, Institute \\ of Neuroscience and Physiology, Sahlgrenska Academy, University of Gothenburg, Gothenburg, \\ Sweden; ${ }^{5}$ University Children's Hospital, Paediatric Endocrinology, Tübingen, Germany; ${ }^{6}$ Unidade \\ de Endocrinologia do Desenvolvimento (LIM42), Hospital das Clinicas da Faculdade de Medicina da \\ Universidade de Sao Paulo, Sao Paulo, Brazil; ${ }^{7}$ Pfizer Inc, Rare Disease, New York 10017; and ${ }^{8}$ Pfizer, \\ Data Management, Sollentuna, Sweden
}

ORCiD numbers: 0000-0003-4890-0262 (A. Dauber); 0000-0002-8211-9237 (M. P. Wajnrajch).

Context: Individual patients vary in their response to growth hormone (GH). No large-scale genome-wide studies have looked for genetic predictors of $\mathrm{GH}$ responsiveness.

Objective: To identify genetic variants associated with GH responsiveness.

Design: Genome-wide association study (GWAS).

Setting: Cohorts from multiple academic centers and a clinical trial.

Patients: A total of 614 individuals from 5 short stature cohorts receiving GH: 297 with idiopathic short stature, 276 with isolated GH deficiency, and 65 born small for gestational age.

Intervention: Association of more than 2 million variants was tested.

Main Outcome Measures: Primary analysis: individual single nucleotide polymorphism (SNP) association with first-year change in height standard deviation scores. Secondary analyses: SNP associations in clinical subgroups adjusted for clinical variables; association of polygenic score calculated from 697 genome-wide significant height SNPs with GH responsiveness.

Results: No common variant associations reached genome-wide significance in the primary analysis. The strongest suggestive signals were found near the B4GALT4 and TBCE genes. After meta-analysis including replication data, signals at several loci reached or retained genomewide significance in secondary analyses, including variants near ST3GAL6. There was no significant association with variants previously reported to be associated with $\mathrm{GH}$ response nor with a polygenic predicted height score.

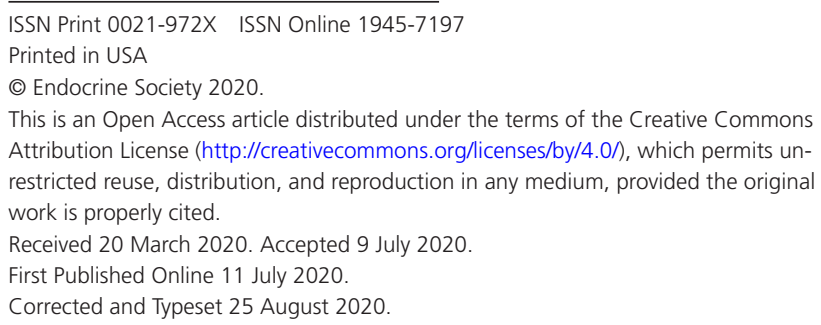

Abbreviations: beta4GalT, beta-1,4-galactosyltransferase; GH, growth hormone;
GHD, GH deficiency; GWAS, genome-wide association study; HE, HumanExome; HO,
Illumina HumanOmni1-Quad BeadChip; HOEE, HumanOmniExpressExome; IGF, insulin-
like growth factor; ISS, idiopathic short stature; MAF, minor allele frequency; MPH,
midparental height; rhGH, recombinant GH; SDS, standard deviation score; SGA, small
for gestational age; SNP, single nucleotide polymorphism. 


\begin{abstract}
Conclusions: We performed the largest GWAS of GH responsiveness to date. We identified 2 loci with a suggestive effect on GH responsiveness in our primary analysis and several genomewide significant associations in secondary analyses that require further replication. Our results are consistent with a polygenic component to $\mathrm{GH}$ responsiveness, likely distinct from the genetic regulators of adult height. (J Clin Endocrinol Metab 105: 3203-3214, 2020)
\end{abstract}

Key Words: growth hormone, pharmacogenetics, short stature, genome-wide association

G rowth disorders resulting in short stature have numerous etiologies, including many different inherited clinical syndromes. Specific genetic etiologies include disorders of the growth hormone-insulinlike growth factor-1 (GH/IGF-1) axis-GH deficiency (GHD) and hormone resistance-and a wide variety of additional genetic syndromes such as Turner syndrome, Noonan syndrome, and skeletal dysplasias (1-3). Other children with short stature are born small for gestational age (SGA) and do not have "catch-up" growth. Finally, the majority of children with short stature have no known specific cause and are classified as idiopathic short stature (ISS).

Recombinant human GH has been used in pediatric populations to increase height for individuals with many of these conditions. There is substantial variability in growth response to $\mathrm{GH}$, not only across types of growth disorders but also between individuals who share the same general etiology for their short stature (4-6). The variable response seen between different disorders suggests that the sensitivity to treatment is associated with the underlying cause of each condition. However, the additional variability within each disorder indicates that individual factors, including possibly genetic variation, also contribute to $\mathrm{GH}$ responsiveness.

Previously, disease-specific models have been developed to predict a patient's response to GH. These models include multiple clinical variables such as parental heights, age at $\mathrm{GH}$ initiation, degree of short stature, peak GH levels on stimulation testing, GH dose, and birth parameters (7). However, these clinical parameters only partially predict variation in treatment response. Given the inclusion of parental height (a proxy for genetic determinants of height) in prediction models, and the high heritability of height, it is quite plausible that genetic variation could influence response to GH. Furthermore, identifying genetic variants that influence response could shed light on the biological mechanisms underlying the variability in response to GH therapy.

Several studies have proposed candidate variants in single genes within the GH/IGF-1 axis for influencing $\mathrm{GH}$ response. The most widely studied of these is a polymorphism in GHR that deletes exon 3 (GHRd3), which has been associated with response to GH therapy in children with short stature. However, this association has only been seen in small samples and has not been consistently replicated (8-12). Similarly, a polymorphism in the IGFBP-3 promoter has been associated with GH response, but this has not been robustly replicated $(13,14)$. The PREDICT study was designed to take a more comprehensive approach to examining genetic predictors of GH response (15). In that study, a more extensive candidate gene study was performed: 170 patients $(110$ with GHD and 60 with Turner syndrome) underwent genotyping of approximately 1500 single nucleotide polymorphisms (SNPs) in a list of 103 candidate genes related to growth. Additionally, baseline gene expression analysis was performed in a subset of patients. The genotypes and gene expression profiles were then correlated with first-year GH response. The study identified a total of 11 genes associated with GH responsiveness in patients with GHD and 10 genes in patients with Turner syndrome. However, a subsequent replication study was performed by the same research group in which none of the original SNP signals were reliably replicated (16). There was some suggestive evidence for signals in the SOS1 and INPPL1 genes. Ultimately, they concluded that genotype information did not add much to the predictive capability of clinical parameters in determining GH responsiveness. The PREDICT study did identify gene expression profiles associated with both growth response (15) as well as with increase in IGF-1 levels at 1 month of treatment (17). Using bioinformatics techniques, they were able to identify pathways, such as glucocorticoid response, which are potentially involved in GH responsiveness.

To date, there have be no large genome-wide association studies (GWASs) of GH responsiveness, which would provide a comprehensive assessment of the role of common genetic variation in GH response. Recent GWASs of adult height have identified hundreds of associated common variants (18) and, as suggested by the correlation between parental height and GH response, these may also be candidates for influencing $\mathrm{GH}$ response and as such merit additional attention. Finally, rarer genetic variants influence height, including those responsible for single-gene disorders $(19,20)$, and these may also influence GH response. For example, some genetic syndromes (such as short 
stature homeobox-containing gene deficiency (19)) are characterized by better responses to $\mathrm{GH}$ than others (such as spondyloepiphyseal dysplasia (20)), consistent with the idea that genetic factors may influence $\mathrm{GH}$ response.

To search for genetic variation that affects response to $\mathrm{GH}$, we carried out what is to our knowledge the largest GWAS of response to $\mathrm{GH}$, consisting of a total of 614 children including 276 with idiopathic GHD, 297 children with ISS, and 41 children born SGA (with neither GHD nor ISS). We identify several suggestive candidate polymorphisms for influencing $\mathrm{GH}$ response and also examine the role of height-associated variants on $\mathrm{GH}$ response. Finally, we observe no evidence to support the previously reported association between the GHR-d3 or IGFBP-3 promoter polymorphisms and GH response.

\section{Participants and Methods}

\section{Description of study samples}

The study included 614 children who were treated with GH on the basis of the diagnosis of GHD, SGA, or ISS. Individuals were recruited from 5 cohorts including the Boston Children's Hospital short stature cohort, Barcelona, Sweden, a Pfizer USA clinical trial for patients with ISS, and individuals who were previously collected by KIGS investigators (Pfizer International Growth Database). The majority (504) of these samples are of self-described European ancestry. A replication sample from Brazil consisted of 113 individuals treated with GH for GHD, SGA, or ISS.

The study was approved by the Institutional Review Board of Boston Children's Hospital as well as by local review boards in Barcelona, Sweden, Pfizer USA, and KIGS (Brazil). Written informed consent was obtained from all patients' parents or legal guardians.

All clinical information was abstracted from the available medical records/research databases at the respective centers. For all patients, we collected information on birth weight, gestational age, peak GH value during pretreatment testing for GHD, age and height at GH start, average GH dose, parents' heights, and height at the end of 1 year of GH therapy. Height standard deviation scores (SDSs) were calculated by adjusting for age and gender according to Prader et al (21). Birth weight SDSs were calculated according to Niklasson et al (22). The midparental height (MPH) SDS was calculated by the following: (father's height SD score + mother's height SD score) $\div 1.61$ (23). Height gain (delta height) was defined by the difference between height SDS at the end of 1 year of treatment and the height SDS at start of GH therapy.

Patients with known chromosomal disorders or other significant comorbid conditions were excluded from the study. We stratified samples by diagnosis. Patients with a peak $\mathrm{GH}$ value below $10 \mathrm{ng} / \mathrm{mL}$ were classified as GHD. Due to the nature of the cohort, GH levels were not assayed centrally but rather were based on each center's reported values. Patients were excluded if they had additional pituitary hormone deficiencies. Patients who had a birth weight SDS below -2 were classified as SGA. Patients could be assigned to both the SGA and GHD categories. Patients who did not meet either of these criteria were classified as ISS. All female patients were required to remain prepubertal throughout the study period, which was defined as Tanner stage 1 breast development. Male participants were required to have testicular volumes $\leq 6 \mathrm{cc}$ or be documented as Tanner stage $\leq 2$ at study completion. The clinical characteristics of the patients are summarized in Table 1.

\section{Genotyping and quality control}

Samples were genotyped using either a combination of the Illumina HumanOmni1-Quad BeadChip (HO) and HumanExome (HE) BeadChip platforms, or on the HumanOmniExpressExome (HOEE) BeadChip v1.0 or v1.2 platforms; all of these platforms cover a similar genome-wide set of common variants and a more comprehensive set of exonic variants. We used Illumina's GenCall algorithm to generate genotype calls for all platforms. For the rarer exonic variants, we generated a second set of genotype calls using zCall (24), which is specifically designed for calling rare SNPs from arraybased platforms.

Quality control was performed separately for each of the platforms (HO, HE, and HOEE). The minimum overall call rate for passing samples was $95 \%$. Samples were also removed for being outliers for amount of heterozygosity, mismatch between reported gender and genotypic data, or close relationship (estimated genomic identity by descent $\geq 0.2$ ) with other samples. Self-reported ancestry and gender were compared with ancestry and sex directly estimated from the genetic data; discordant samples were removed. Standard additional filters were applied to remove poorly genotyped SNPs, including variant call rate $<95 \%$, HardyWeinberg equilibrium $P$ value $<10^{-6}$, and evidence of nonrandom missingness or batch effects. In total, 588 individuals had genotype data passing quality control and the required phenotypic information for association analysis. 


\begin{tabular}{|c|c|c|}
\hline & $\begin{array}{l}\text { GWAS } \\
\text { Cohorts }\end{array}$ & $\begin{array}{l}\text { Replication } \\
\text { Cohort }\end{array}$ \\
\hline Individuals & 614 & 113 \\
\hline Male & 437 & 73 \\
\hline Female & 177 & \\
\hline $\begin{array}{l}\text { Gestational age } \\
\text { (weeks) }\end{array}$ & $38.6(2.9)$ & $37.2(3.1)$ \\
\hline Birth weight (g) & 2969 (693) & $2754(809)$ \\
\hline Birth weight SDS & $-0.70(1.14)$ & $-0.60(1.48)$ \\
\hline $\begin{array}{l}\text { Midparental height } \\
(\mathrm{cm})\end{array}$ & $164.6(5.8)$ & $161.9(5.35)$ \\
\hline Age at start (years) & $8.14(2.71)$ & $8.31(2.55)$ \\
\hline $\begin{array}{r}\text { Average growth } \\
\text { hormone dose } \\
\text { (mg/kg/week) }\end{array}$ & $0.28(0.12)$ & $0.29(0.06)$ \\
\hline $\begin{array}{l}\text { Height at start (Prader } \\
\text { SDS) }\end{array}$ & $-3.00(0.72)$ & $-3.77(1.18)$ \\
\hline $\begin{array}{l}\Delta \text { height SDS during } \\
\text { first year of therapy }\end{array}$ & $0.78(0.40)$ & $0.76(0.72)$ \\
\hline ISS & 297 & 42 \\
\hline GHD & 276 & 44 \\
\hline SGA & 65 & 30 \\
\hline SGA \& GHD & 24 & 3 \\
\hline
\end{tabular}

The numbers in parens are SD, standard deviations. The "SDS" listed for Birth Weight SDS, Prader SDS and Height SDS are standard deviation scores.

Abbreviations: GHD: growth hormone deficiency; GWAS, genomewide association study; ISS, idiopathic short stature; SDS: standard deviations above or below the mean; SGA: small for gestational age; SGA_SDS, standard deviation score for being small for gestational age.

\section{Genotype imputation}

To expand the coverage of the genotyping platforms to include the vast majority of common variants, we imputed genotypes for all polymorphic markers in the 1000 Genomes Phase I Integrated Release Version 3 (March 2012) (25), using the program IMPUTE2 (26, 27), thereby generating datasets of 36388217 autosomal and 1250157 sex chromosomal variants. The data from $\mathrm{HO}$ and $\mathrm{HE}$ platforms were merged and then imputed; the remaining samples genotyped on the HOEE platform were imputed separately. We removed SNPs with a low quality of imputation (info score $<0.4$ ).

\section{Association analysis}

The primary outcome was the change in height SDS; we performed an inverse normal transformation to ensure normality of the outcome variable, but results were similar if this transformation was omitted. We carried out association testing between this outcome and SNP genotype (coded as allele dosage between 0 and 2), using an additive model in a regression framework implemented in PLINK (28). We performed analyses stratified by diagnosis (all diagnoses, ISS only, and GHD only; the number of SGA samples was too small for reliable analyses) and by ancestry (all ancestries and European ancestry only). For each analysis, we used 2 different models: a minimally adjusted model (age and gender included as covariates) and a maximally adjusted model (age; gender; gestational age; birth weight SDS; age at start of GH therapy; GH dosage; height SDS at start of $\mathrm{GH}$ therapy; $\mathrm{MPH}$; and whether the patient has mild or severe GHD, defined as a peak GH level $<5$, included as covariates). In addition, principal components (29) were included as covariates to account for ancestry; for the multiethnic analysis, 10 principal components were included; for European samples, 3 principal components were included. The primary analysis was considered to be all ancestries, all diagnoses, and a minimally adjusted model (Fig. 1).

To account for the potential confounding effect due to different genotyping platforms, the analyses were performed separately for the Pfizer USA samples genotyped on $\mathrm{HO} / \mathrm{HE}$ and all other samples genotyped on HOEE, and results were meta-analyzed. For meta-analysis, we used the inverse-variance fixed effects method to combine the results, using METAL (30), weighed by standard error. Genome-wide significance for our primary analysis was set at $P<5 \times 10^{-8}$.

\section{Association analysis of low-frequency coding variants}

For rarer (minor allele frequency $<5 \%$ ) coding variants represented on the exome chip, 2 sets of analyses were performed using rare variant tests (31). We performed association analysis of each variant individually (single-variant analysis) and aggregate analysis of missense, splice, and loss-of-function variants within each gene (gene-based analysis). Gene-based analyses were performed with sequence kernel association tests (32), and meta-analysis of results from the 2 genotyping platforms were performed using RAREMETAL (33).

For single-variant exome analysis, we set the significance threshold for single-variant association analysis as $P<6.93 \times 10^{-7}$ (Bonferroni correction for 72171 polymorphic variants) for analysis of samples with $\mathrm{GH}$ responsiveness. The significance threshold for a genebased test association as $P<2.5 \times 10^{-6}$ (Bonferroni correction for 20000 genes). The analyses were carried out under the same phenotypic models as for GWAS.

\section{Association analysis of previously reported height- associated variants}

For each individual, we calculated a genetic polygene score (34) as implemented in PLINK (28), using 697 height-associated loci identified by the GIANT consortium (18). Specifically, the reported regression coefficient of each SNP on height and the individual genotype at each SNP is used to create a weighted polygene score 


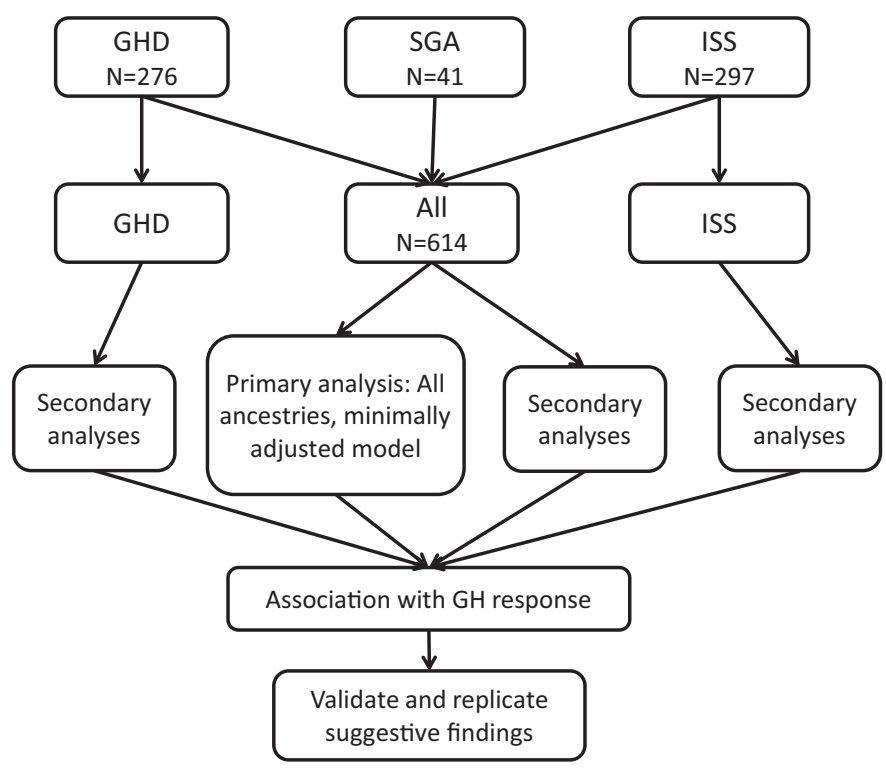

Figure 1. Analysis plan for testing association to growth hormone response. The primary analysis was to combine patients in all 3 categories: growth hormone deficiency (GHD), small for gestational age (SGA) and idiopathic short stature (ISS), including all ancestries and performing a minimally adjusted analysis (age, sex, and principal components of genetic ancestry). Secondary analyses included testing GHD or ISS alone, limiting the analyses to individuals of European ancestry and adjusting for additional covariates. Additional covariates were gestational age in weeks, birthweight standard deviation score (SDS), age at GH start, average GH dose, height SDS (Prader) before treatment, average height of parents, and severity of GHD. For principal components of ancestry, the top 10 eigenvalues were used for all ancestry analyses and the top 3 eigenvalues for analyses of European ancestry samples. For single-variant analysis, associations were considered to have genome-wide significance if they reached $P<5 \times 10^{-8}$ in the primary analysis; suggestive associations were those that reached $P<5 \times 10^{-7}$ in the primary or secondary analyses.

for each individual. We calculated the variance in $\mathrm{GH}$ response explained by this polygene score (adjusted $\mathrm{R}^{2}$ estimated from a linear regression model incorporating the score as the predictor and the normalized height SDS as an outcome, adjusting for age at $\mathrm{GH}$ initiation and gender).

\section{Association analysis of GHR-d3 and IGFBP3 variants}

We tested for association with variants previously reported to be associated with $\mathrm{GH}$ response, using the methods described above. For the GHR-d3 variant (10), we used as a proxy the SNP rs6873545 $\left(r^{2}\right.$ between GHR-d3 and rs6873545=1 in the HapMap CEU European ancestry sample); we imputed the previously reported IGFBP3 rs2854744 variant.

\section{Validation and replication of variants with suggestive associations to $\mathrm{GH}$ response}

We used PLINK to "clump" variants into loci based on linkage disequilibrium $\left(r^{2}>0.5\right)$, with a single lead variant in each locus selected based on the best $P$ value. We selected for additional genotyping 3 variants that had associations $P<5 \times 10^{-7}$ in the primary analysis and 5 additional variants with $P<5 \times 10^{-8}$ in any secondary analysis, as well as an additional 29 variants with associations $P<5 \times 10^{-7}$ in any secondary analysis. We performed Sanger sequencing in a subset of the GWAS samples to confirm good agreement with the original GWAS data before proceeding to genotyping. In total, we obtained genotype data in the Brazilian replication sample for 26 of these 37 SNPs; 5 of the 28 SNPs were monomorphic in the replication sample and were not analyzed further. Genotyping was performed using the Sequenom MassArray platform as previously described (35) or, for a subset of variants that could not be genotyped successfully, by Sanger sequencing.

\section{Results}

To search for genetic determinants of response to $\mathrm{GH}$, we studied 614 children treated with GH and assembled from several studies (Table 1; see Methods). The participants were categorized as having a diagnosis of GHD $(\mathrm{N}=276)$, SGA $(\mathrm{N}=41)$, or ISS $(\mathrm{N}=297)$. The majority $(\mathrm{N}=504)$ of these samples were of European ancestry. The primary outcome in our study was the increment in height SDS during the first year of therapy. We first performed a GWAS and imputed $(26,27)$ genotypes for all polymorphic variants with frequency $>1 \%$ in the 1000 Genomes Phase I Integrated Release Version 3 (March 2012) (25). Our primary analysis included all samples, regardless of diagnosis, and was adjusted for age, sex, and principal components of genetic ancestry (Fig. 1; see Methods). We also performed secondary analyses stratified by ancestry and diagnosis, adjusting for 
a more extensive set of covariates (maximally adjusted; see Methods). The distributions of test statistics for each analysis were not significantly inflated: genomic inflation correction factors $\left(\lambda_{\mathrm{GC}}\right)$ ranged between 1.004 and 1.021, indicating minimal systematic bias (Fig. 2).

\section{GWAS analysis of common variants with GH response}

We first tested common variants with minor allele frequency (MAF) above 1\% for association with $\mathrm{GH}$ response. In our primary analysis (Fig. 1), no variants achieved genome-wide significance $\left(P<5 \times 10^{-8}\right.$; Table 2). The strongest suggestive $\left(P<5 \times 10^{-7}\right)$ associations with $\mathrm{GH}$ response were observed with a common variant near the B4GALT4 gene: rs7628585 (intronic, MAF $\left.=0.182 ; P=5.54 \times 10^{-8}\right) ;($ Fig. 3$)$ and with a common SNP in the TBCE gene, rs1977748 (intronic, $\left.\mathrm{MAF}=0.411 ; P=3.75 \times 10^{-7}\right)$.

In secondary analyses, $5 \mathrm{SNPs}$ had association $P$ values below $5 \times 10^{-8}$ (Table 2); all of these had minor allele frequencies below $5 \%$. In light of the sample size of this study, these associations, especially those specific to 1 diagnosis, should be viewed as tentative in the absence of additional replication. A low-frequency intronic SNP ( $\mathrm{rs} 55704135, \mathrm{MAF}=3 \%$ ) in the UBE4B gene showed the strongest association to $\mathrm{GH}$ response in the analysis of all European ancestry individuals under the maximally adjusted model $\left(P=4.2 \times 10^{-9}\right)$. An intergenic SNP $(r s 189532746$, frequency $=1.3 \%)$ between CPOX

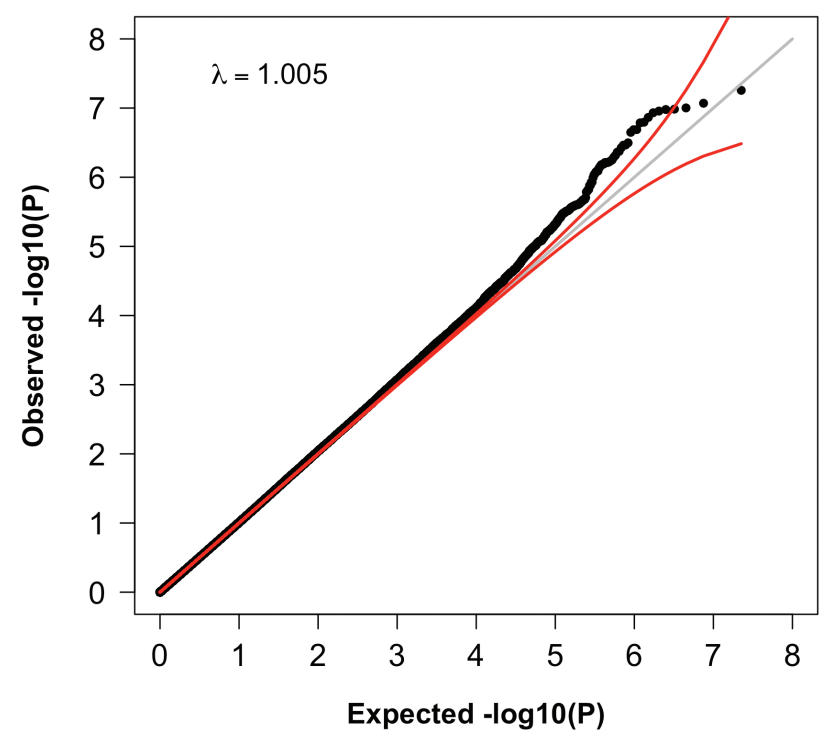

Figure 2. Quantile-quantile (QQ) plot for results from the primary association analysis of common variants with growth hormone response. Each point represents the result from a single variant (genotyped or imputed), ranked by observed $P$ value. The $x$-axis indicates the expected $P$ value and the y-axis indicates the observed $P$ value, both in negative log scales. The gray line is the expected result under the null, and the red lines indicate 95\% confidence intervals around the expectation. and ST3GAL6 showed its strongest association in the analysis of individuals with ISS of all ancestries under the minimally adjusted model $\left(P=6.95 \times 10^{-9}\right)$. An intergenic SNP (rs78263566, frequency $=2.5 \%$ ) between CLEC7A and OLR1 and an intronic SNP $($ rs 74523128, frequency $=1.0 \%)$ in LAPTM4B showed strongest associations in the analyses of individuals with GHD under the maximally adjusted model in European ancestry individuals $\left(P=8.5 \times 10^{-9}\right.$ and $\left.P=1.5 \times 10^{-8}\right)$. Finally, a low-frequency intronic SNP (rs144751704, MAF $=1.4 \%$ ) located in the NT5DC1 gene and upstream of COL10A1 showed the strongest association to GH response in the analysis of all European ancestry individuals under the minimally adjusted model $\left(P=2.1 \times 10^{-8}\right)$. We also identified 29 additional variants with $P<5 \times 10^{-7}$ in secondary analyses, a level of significance suggestive of association.

\section{Attempted replication of potential signals of association with response to recombinant $\mathrm{GH}$}

We first focused on the 2 signals that reached suggestive significance in our primary analysis: rs7628585 near B4GALT4 and rs1977748 near TBCE. Neither of these signals were replicated nor reached genome-wide significance (Table 2). We also attempted to replicate the 5 signals that had reached genome-wide significance in a secondary analysis, meta-analyzing the discovery and replication samples using either the same model for which we observed the best evidence for association in the discovery sample or using the primary analysis (minimal covariates, all diagnoses) to maximize power. Two signals for which we were able to obtain replication data retained genome-wide significance after meta-analysis (rs55704135 near $U B E 4 B$; meta-analysis $P=2.65 \times 10^{-8}$ in the European ancestry/all diagnoses/maximum covariate analysis, and rs78263566 near CLEC7A; meta-analysis $P=1.67 \times 10^{-8}$ in the European ancestry/ GHD/maximum covariate analysis, Table 2). However, for both of these signals, the evidence for association decreased after replication, so further validation of these associations would be needed. Two other genome-wide significant variants, (rs189532746, between CPOX and ST3GAL6, and rs78263566, between CLEC7A and OLR1) were not polymorphic in the replication analysis, and we were unable to successfully genotype the fifth signal (rs144751704, located in NT5DC1 and upstream of COL10A1) in the replication sample.

We also attempted to replicate 29 signals with suggestive signals in secondary analyses. Of the 19 signals that were successfully genotyped, 12 were polymorphic in the replication sample, and 2 achieved genomewide significance in meta-analysis with the replication data when analyzed under the same model as was 


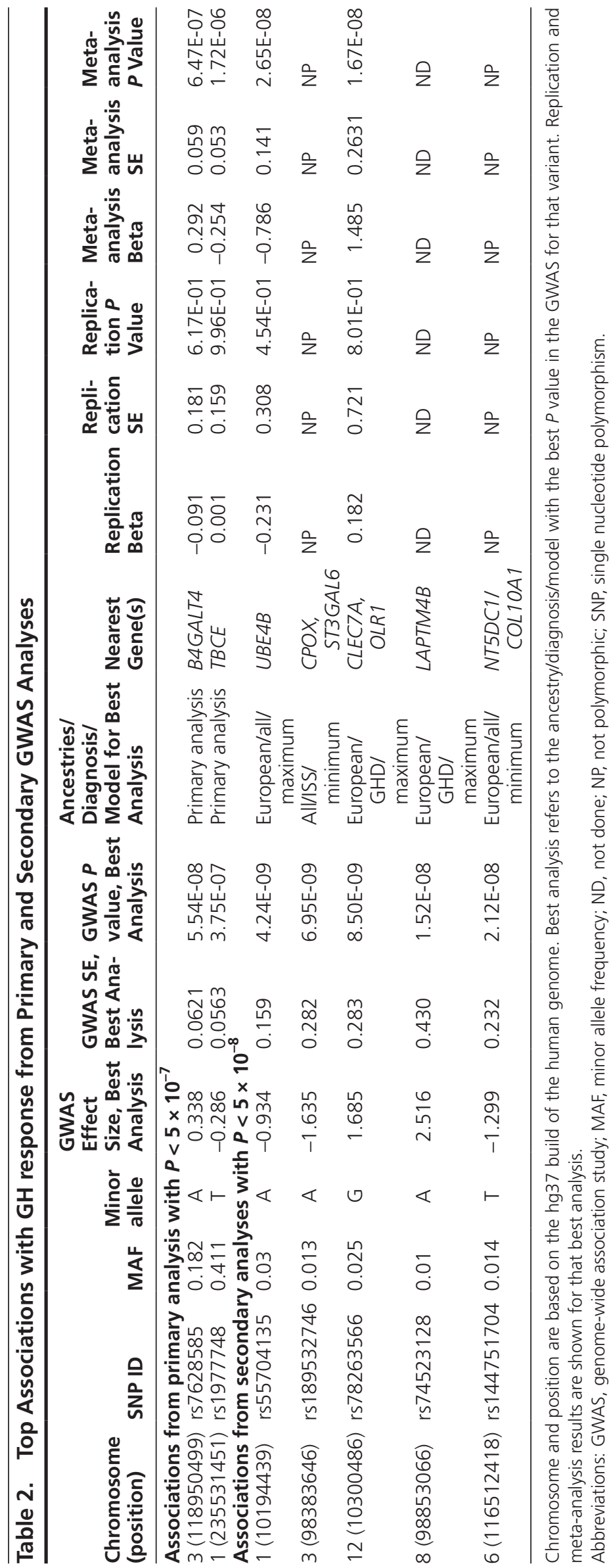




\section{rs7628585}

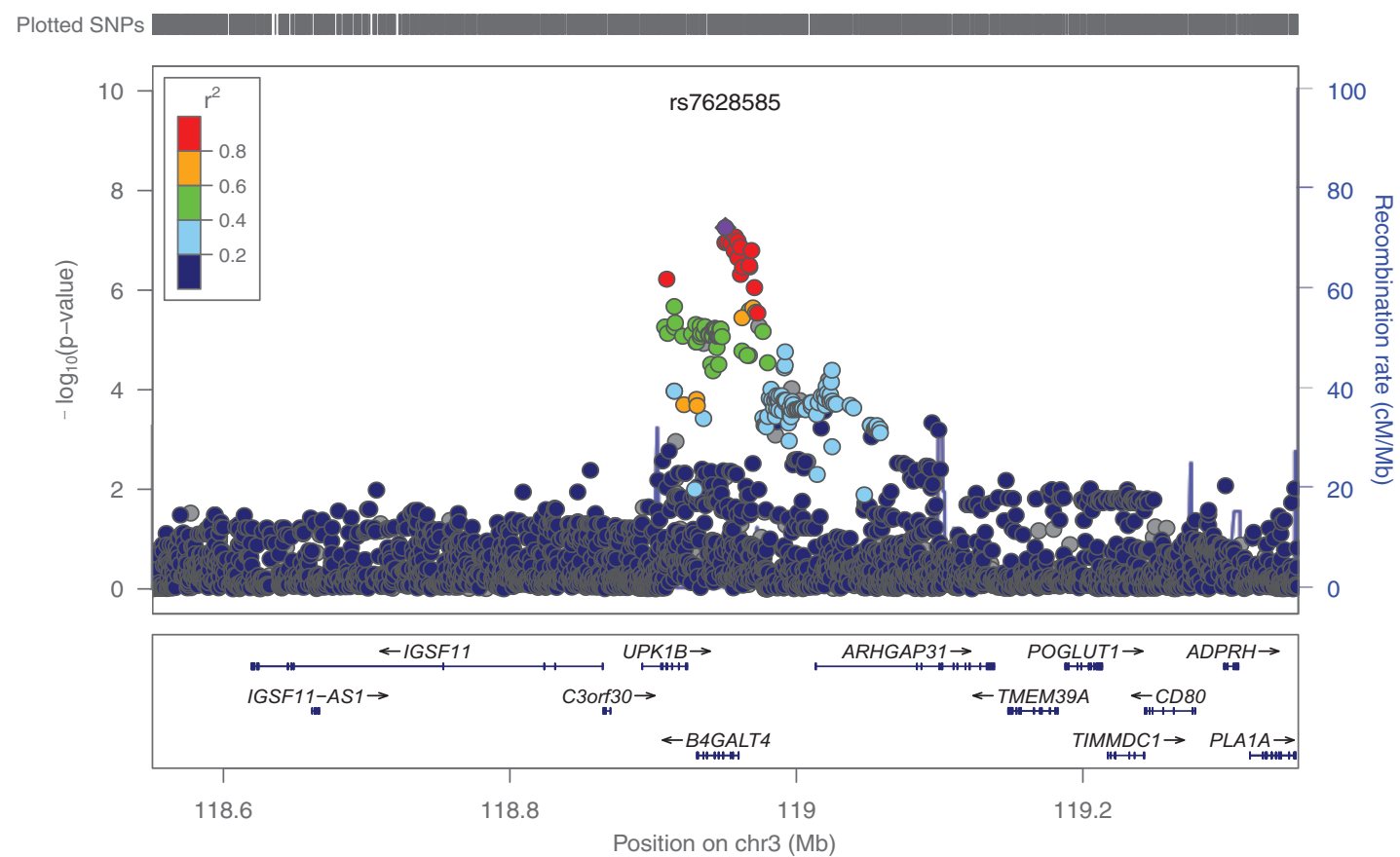

Figure 3. LocusZoom plot for the B4GALT4 locus. The x-axis indicates position on chromosome 3 surrounding the lead signal of association near B4GALT4, rs7628585. Genes depicted in their respective locations. Each dot is a variant in the region tested and is colored by its correlation ( $r 2$ ) with rs7628585 according to the color key. Blue lines indicate recombination rates. The arrow and circle indicate the location and association signal of the second signal at B4GALT4, rs35583194; this insertion/deletion variant was not included in the LocusZoom plot because it is not present in the 1000 Genomes reference panel. SNP, single nucleotide polymorphism.

highlighted by the discovery sample. These 2 signals were rs75922185 near MGAT5; meta-analysis $P=4.93 \times 10^{-8}$ in the European ancestry/all diagnoses/maximum covariate analysis, and rs151058087 near AEBP2; meta-analysis $P=1.67 \times 10^{-8}$ in the all ancestry/ISS/ maximum covariate analysis (Online Supplementary Table 1) (36). The variant near AEBP2 was the only one to achieve nominal significance in the replication sample. Of potential interest, rs115307564 near $R B M S 3$, strongly replicated under the primary analysis model (replication $P=7.96 \times 10^{-4}$, meta-analysis $P=1.67 \times 10^{-6}$; Online Supplementary Table 1) (36). This variant therefore represents another promising signal of association.

\section{Association analysis of rarer coding variation with response to recombinant $\mathrm{GH}$ using exome array genotypes}

To determine the role of low-frequency coding variants, we also analyzed directly genotyped coding SNPs present on the exome array platforms. In single-variant analysis, no SNPs were significant after correcting for multiple testing (see Methods). Because single-variant tests are often underpowered to detect associations with rare variants, we also carried out gene-based association tests using missense, splice, and loss-of-function variants within each gene (see Methods), combining information from the different variants in each gene using the sequence kernel association test (32), and limiting analysis to variants below $1 \%$. No genes were significant after correcting for multiple testing (see Methods). A full set of association results for our primary analysis for all variants with MAF above $1 \%$ and all directly genotyped coding variants will be made available.

\section{GWAS analysis of variants previously associated with GH response or adult height}

We also focused specifically on candidate polymorphisms previously associated with response to $\mathrm{GH}$, including the $G H R \mathrm{~d} 3$ variant $(10-12)$, which is represented in our study by the highly correlated SNP rs6873545 (37), as well as the rs 2854744 polymorphism in the IGFBP-3 promoter $(13,38)$. We confirmed that the rs6873545 was a good proxy for the GHR d3 variant in our study (see Methods). We observed no evidence at either of these variants for association with response to $\mathrm{GH}(P>0.05$ under all models). Thus, we could not replicate the previous associations.

We also tested a hypothesis that genetic predisposition to tall or short stature could influence response to GH. For each individual, we constructed a genetic polygene score based on the previously established 697 
height variants and tested for association between this polygene score and the response to $\mathrm{GH}$. We did not see even nominally significant evidence of association, suggesting that genetic control of final height and response to $\mathrm{GH}$ are not strongly connected.

\section{Discussion}

We report what is, to our knowledge, the largest GWAS of response to $\mathrm{GH}$ to date. We leveraged the large sample size to interrogate both a primary analysis (all samples, with a minimal set of covariates) as well as several secondary analyses, including testing for association in all samples and in European samples only, with specific diagnoses (ISS and GHD), and adjusting for a larger set of known clinical covariates (gestational age, age at start of GH therapy, GH dosage, height SDS at start of GH therapy, MPH).

We detected 37 suggestive associations $\left(P<5 \times 10^{-7}\right)$ from this GWAS, including 3 suggestive associations from our primary analysis and 5 that had $P$ values below $5 \times 10^{-8}$ in at least 1 secondary analysis. The strongest of the associations in the primary analysis was with common variation near the B4GALT4 and TBCE genes. However, despite these strongly suggestive genetic data, we did not observe associations with GH response in our replication sample. Possible explanations for the lack of replication include lack of power in the replication sample, differences in ethnicity (and hence different levels of linkage disequilibrium with an untyped causal variant), or that both of these suggestive associations are statistical fluctuations rather than true associations. Interestingly, the B4GALTT4 gene is 1 of 7 beta-1,4galactosyltransferase (beta4GalT) genes, and mutations in B4GALT7 lead to abnormal skeletal growth (39). However, mutations in B4GALT4 have not yet been reported to be associated with any specific disease. Of interest, a low-frequency variant that reached genomewide significance in a secondary analysis is located near ST3GAL6, a sialyltransferase that acts in the same pathway as the beta4GalT genes. The frequency of this variant was too low to robustly perform replication.

Of the remaining variants that we were able to assay in the replication sample (see Methods), 4 signals had meta-analysis $P$ values that reached or retained genomewide significance in secondary analysis (near $U B E 4 B$, CLEC7A, MGAT5, and AEBP2). Remarkably, MGAT5 also encodes a glycosyltransferase, although not obviously in the same pathway as the beta4GalT genes. Thus, our results provide an intriguing suggestion that variation in glycosylation pathways may regulate the response to $\mathrm{GH}$.
Another low-frequency variant, near RBMS3, was the only one to show a strong signal in the replication cohort primary analysis but did not reach genomewide significance. RBMS3 encodes a long noncoding ribonucleic acid that may modulate signaling by transforming growth factor beta, a well-known modulator of growth; of note, the TGFBR2 gene is adjacent to RBMS3. This variant, as well as the variants described in the previous paragraphs, represents signals that would benefit from further validation to see if they are robustly associated with GH response.

We also tested variants previously associated with response to recombinant $\mathrm{GH}$ (rhGH), including the exon 3 deletion variant in GHR (10-12) and the rs2854744 and rs924140 variants in IGFBP3 $(13,38)$. We found no evidence of association with response to rhGH for any of these variants (all $P>0.05)$. One possibility why we failed to see an association in our large sample is that the findings from the prior studies are false-positives. We note that our study population is a heterogeneous collection of patients with short stature, so differences in patient population could also explain these discrepant findings. However, we also saw no evidence of association when we restricted our analysis to patients with ISS or to those with GHD.

We also tested whether the 697 height-associated loci are associated with response to rhGH. We found no strong association with GH response for any heightassociated loci, either individually or jointly. One possible explanation is that the $\mathrm{GH}$ response is regulated by different biological mechanisms than for height; another possible explanation is that variation in $\mathrm{GH}$ response is associated with variants that are rare and have not been identified from previous GWAS analyses. Comprehensive sequencing of samples with data on $\mathrm{GH}$ response could help address these possibilities. For example, if a fraction of patients treated with GH have rare, nearly mendelian causes of short stature, then the variable response to $\mathrm{GH}$ could be partly dictated by the variable underlying etiologies in these patients.

Limitations of our study include the collection of samples from various locations in the world with various ethnic backgrounds, and a mixture of diagnoses. We addressed this heterogeneity by performing secondary analyses of more homogeneous, if smaller, subgroups of individuals. The sample size is larger than previous studies but still quite small as compared with many GWAS studies and, therefore, has limited power to detect variants associated with GH response. A sample size of 614 should have $80 \%$ power to detect associations that explain, in our heterogeneous population, $6.5 \%$ of the variance in $\mathrm{GH}$ response. Although 
effect sizes this large are unusual for polygenic traits, large effect sizes have been seen in other pharmacogenomics scenarios. Thus, any heritable contribution to $\mathrm{GH}$ response is likely polygenic. While our work shows that there are likely no common variants that on their own have clinically significant predictive power, larger sample sizes would be needed to identify variants with smaller effects that in combination, as part of a polygenic risk score, could still provide meaningful prediction of the response to $\mathrm{GH}$.

Our study only examined the relationship between genotype and growth response. As noted earlier, other approaches, such as gene expression profiling, can look at other molecular predictors of $\mathrm{GH}$ response, and these alternate approaches have been reviewed elsewhere (40). For example, 1 study looked at the methylation status of the IGF-1 promoter and found that approximately $25 \%$ of the first-year GH response may be attributable to differences in methylation at the P2 promoter of IGF-1 (41). Additionally, while our study focused on first-year height response, consideration must be given to other definitions of $\mathrm{GH}$ responsiveness including biochemical, transcriptomic, and proteomic responses, which may offer more mechanistic insights into GH biology.

In summary, we have completed the largest genetic study to date of response to GH. We were unable to replicate previous associations, nor do we identify any new variants that are clearly and robustly associated with GH response. However, some associations reached genome-wide significance in secondary analyses and merit further investigation, and our data collectively raise the hypothesis that variation in glycosylation may contribute to variation in $\mathrm{GH}$ response. Larger sample sizes will be needed to more definitively identify any genetic factors that robustly influence the response to GH.

\section{Acknowledgments}

This study was supported by Pfizer.

Samples from patients with ISS came from Pfizer-sponsored study A6281280, with the following investigators in the United States: Moris Angulo; Yong Bao; Deborah Bowlby; Dennis Brenner; Rosalind Brown; Mauri Carakushansky; Joseph Cernich; Jay Cohen; Debra Counts; Paul Desrosiers; Oscar Escobar; Myrto Frangos; Graeme Frank; Sherry L. Franklin; Mitchell Geffner; Michele Hutchison; Stephen Kemp; Lynne Levitsky; Revi Mathew; Sunil Nayak; Robin Nemery; Ron Newfield; Richard Noto; Leslie Plotnick; Teresa Quattrin; Robert Rapaport; Edward Reiter; David Repaske; Samuel Richton; Judith Ross; Deborah Rotenstein; Ellen Sher; Lawrence Silverman; Michael Tansey; Svetlana Ten; Paul Thornton; Sharon Travers; Nancy Wright; Ping Zhou; William Zipf.
Spanish patients were recruited in Hospital Universitari Vall d'Hebron and Vall d'Hebron Institut de Recerca (VHIR).

Swedish patients were recruited from clinical trial TRN 88-080, which was initiated in 1988 as a Kabi-sponsored study and changed to an Investigator-sponsored study in the 1990s, with K.A.W. as sponsor. The principle investigator was Kerstin Albertsson-Wikland and co-investigators included: A. Stefan Aronsson, Jan Gustafsson, Lars Hagenäs, Sten A. Ivarsson, Berit Kriström, Claude Marcus, Karl Olof Nilsson, Martin Ritzén, Torsten Tuvemo, Otto Westphal, Jan Åman.

KIGS Investigators: Jean de Schepper (country coordinator), Vrije Universiteit Brussels (VUB), Brussels; Marine Cools, Ghent University, Ghent; Jan Lebl, Motol University Hospital, Prague; Jana Černá, FN Ostrava State Hospital, Ostrava; Michael B. Ranke and Gerhard Binder, University Children's Hospital, Pediatric Endocrinology and Diabetology, Tübingen; Helmuth Dörr, University Children's Hospital, Pediatric Endocrinology and Diabetology, Erlangen; Berthold Hauffa, Paediatric Endocrinology, Department Paediatrics, University Hospital of Essen, Essen; Heinrich Schmidt, Dr von Haunersches Kinderspital, München; Franziska Lorenzen, Pediatric Endocrinology Practice, Hannover; Roland Pfäffle, University of Leipzig Hospitals and Clinics, Leipzig; Klaus Mohnike, Otto von Guericke University Magdeburg, Magdeburg; Lohtar Reinken, Evangelisches Krankenhaus, Hamm; Bernhard Köster, Klinikum, Lüdenscheid; Ludmila Kostalova, Comenius University in Bratislava and University Children's Hospital Bratislava, Limbova 1, Bratislava.

\section{Additional Information}

Correspondence and Reprint Requests: Michael Wajnrajch, MD MPA, Endocrine Care \& Inborn Errors of Metabolism, Pfizer Inc, 235 East $42^{\text {nd }}$ Street, MS 235-10-01, New York, NY 10017, USA. E-mail: Michael.Wajnrajch@Pfizer.com.

Disclosure Summary: A.D. has received consulting fees from OPKO Biologics, Sandoz, Novo Nordisk, Ipsen, and Pfizer; A.A.L.J. received speaker fees from Sandoz and NovoNordisk; K.A.W. received an unrestricted grant from Pfizer; M.B.R. has received speaker fees from Novo Nordisk, Merck Serono, Sandoz and Mediagnost. J.C., M.P.W., and C.C.H. are employees of Pfizer Inc; A.L. was an employee of Pfizer Health AB; Y.M. is now an employee of Fulgent Genetics; J.N.H. received research support from Pfizer Inc for this study and is on the scientific advisory board of Camp4 Therapeutics; S.V., B.W., L.A., and A.C. have nothing to declare.

Data Availability: The datasets generated during and/or analyzed during the current study are not publicly available but are available from the corresponding author on reasonable request.

\section{References}

1. Guo MH, Shen Y, Walvoord EC, et al. Whole exome sequencing to identify genetic causes of short stature. Horm Res Paediatr. 2014;82(1):44-52.

2. David A, Hwa V, Metherell LA, et al. Evidence for a continuum of genetic, phenotypic, and biochemical abnormalities 
in children with growth hormone insensitivity. Endocr Rev. 2011;32(4):472-497.

3. Seaver LH, Irons M; American College of Medical Genetics (ACMG) Professional Practice and Guidelines Committee. ACMG practice guideline: genetic evaluation of short stature. Genet Med. 2009;11(6):465-470.

4. Richmond E, Rogol AD. Current indications for growth hormone therapy for children and adolescents. Endocr Dev. 2010;18:92-108.

5. Rosenbloom AL. Recombinant human insulin-like growth factor I (rhIGF-I) and rhIGF-I/rhIGF-binding-protein-3: new growth treatment options? J Pediatr. 2007;150(1):7-11.

6. Ranke MB, Lindberg A, Mullis PE, et al. Towards optimal treatment with growth hormone in short children and adolescents: evidence and theses. Horm Res Paediatr. 2013;79(2):51-67.

7. Ranke MB, Lindberg A. Predicting growth in response to growth hormone treatment. Growth Horm IGF Res. 2009;19(1):1-11.

8. Wassenaar MJ, Dekkers OM, Pereira AM, et al. Impact of the exon 3-deleted growth hormone $(\mathrm{GH})$ receptor polymorphism on baseline height and the growth response to recombinant human GH therapy in GH-deficient (GHD) and non-GHD children with short stature: a systematic review and meta-analysis. J Clin Endocrinol Metab. 2009;94(10):3721-3730.

9. Carrascosa A, Esteban C, Espadero R, et al.; Spanish SGA Study Group. The d3/fl-growth hormone $(\mathrm{GH})$ receptor polymorphism does not influence the effect of GH treatment $(66$ microg/kg per day) or the spontaneous growth in short non-GH-deficient smallfor-gestational-age children: results from a two-year controlled prospective study in 170 Spanish patients. J Clin Endocrinol Metab. 2006;91(9):3281-3286.

10. Dos Santos C, Essioux L, Teinturier C, Tauber M, Goffin V, Bougnères $\mathrm{P}$. A common polymorphism of the growth hormone receptor is associated with increased responsiveness to growth hormone. Nat Genet. 2004;36(7):720-724.

11. Binder G, Baur F, Schweizer R, Ranke MB. The d3-growth hormone $(\mathrm{GH})$ receptor polymorphism is associated with increased responsiveness to $\mathrm{GH}$ in Turner syndrome and short small-for-gestational-age children. J Clin Endocrinol Metab. 2006;91(2):659-664.

12. Binder G, Trebar B, Baur F, Schweizer R, Ranke MB. Homozygosity of the $\mathrm{d} 3$-growth hormone receptor polymorphism is associated with a high total effect of GH on growth and a low BMI in girls with Turner syndrome. Clin Endocrinol (Oxf). 2008;68(4):567-572.

13. Costalonga EF, Antonini SR, Guerra-Junior G, Mendonca BB, Arnhold IJ, Jorge AA. The -202 A allele of insulin-like growth factor binding protein-3 (IGFBP3) promoter polymorphism is associated with higher IGFBP-3 serum levels and better growth response to growth hormone treatment in patients with severe growth hormone deficiency. J Clin Endocrinol Metab. 2009;94(2):588-595.

14. van der Kaay DC, Hendriks AE, Ester WA, et al. Genetic and epigenetic variability in the gene for IGFBP-3 (IGFBP3): correlation with serum IGFBP-3 levels and growth in short children born small for gestational age. Growth Horm IGF Res. 2009;19(3):198-205.

15. Clayton $\mathrm{P}$, Chatelain $\mathrm{P}$, Tatò L, et al. A pharmacogenomic approach to the treatment of children with GH deficiency or Turner syndrome. Eur J Endocrinol. 2013;169(3):277-289.

16. Stevens A, Murray P, Wojcik J, et al.; PREDICT Investigator Group. Validating genetic markers of response to recombinant human growth hormone in children with growth hormone deficiency and Turner syndrome: the PREDICT validation study. Eur J Endocrinol. 2016;175(6):633-643.

17. Stevens A, Clayton P, Tatò L, et al. Pharmacogenomics of insulinlike growth factor-I generation during $\mathrm{GH}$ treatment in children with GH deficiency or Turner syndrome. Pharmacogenomics J. 2014;14(1):54-62.
18. Wood AR, Esko T, Yang J, et al.; Electronic Medical Records and Genomics (eMEMERGEGE) Consortium; MIGen Consortium; PAGEGE Consortium; LifeLines Cohort Study. Defining the role of common variation in the genomic and biological architecture of adult human height. Nat Genet. 2014;46(11):1173-1186.

19. Blum WF, Ross JL, Zimmermann AG, et al. GH treatment to final height produces similar height gains in patients with SHOX deficiency and Turner syndrome: results of a multicenter trial. J Clin Endocrinol Metab. 2013;98(8):E1383-E1392.

20. Kanazawa H, Tanaka H, Inoue M, Yamanaka Y, Namba N, Seino Y. Efficacy of growth hormone therapy for patients with skeletal dysplasia. J Bone Miner Metab. 2003;21(5):307-310.

21. Prader A, Largo RH, Molinari L, Issler C. Physical growth of Swiss children from birth to 20 years of age. First Zurich longitudinal study of growth and development. Helv Paediatr Acta Suppl. 1989;52:1-125.

22. Niklasson A, Albertsson-Wikland K. Continuous growth reference from $24^{\text {th }}$ week of gestation to 24 months by gender. BMC Pediatr. 2008;8:8.

23. Cole TJ. Some questions about how growth standards are used. Horm Res. 1996;45(Suppl 2):18-23.

24. Goldstein JI, Crenshaw A, Carey J, et al.; Swedish Schizophrenia Consortium; ARRA Autism Sequencing Consortium. zCall: a rare variant caller for array-based genotyping: genetics and population analysis. Bioinformatics. 2012;28(19): 2543-2545.

25. Auton A, Brooks LD, Durbin RM, et al.; 1000 Genomes Project Consortium. A global reference for human genetic variation. Nature. 2015;526(7571):68-74.

26. Marchini J, Howie B, Myers S, McVean G, Donnelly P. A new multipoint method for genome-wide association studies by imputation of genotypes. Nat Genet. 2007;39(7):906-913.

27. Howie BN, Donnelly P, Marchini J. A flexible and accurate genotype imputation method for the next generation of genome-wide association studies. PLoS Genet. 2009;5(6):e1000529.

28. Purcell S, Neale B, Todd-Brown K, et al. PLINK: a tool set for whole-genome association and population-based linkage analyses. Am J Hum Genet. 2007;81(3):559-575.

29. Price AL, Patterson NJ, Plenge RM, Weinblatt ME, Shadick NA, Reich D. Principal components analysis corrects for stratification in genome-wide association studies. Nat Genet. 2006;38(8):904-909.

30. Willer CJ, Li Y, Abecasis GR. METAL: fast and efficient metaanalysis of genomewide association scans. Bioinformatics. 2010;26(17):2190-2191.

31. Huyghe JR, Jackson AU, Fogarty MP, et al. Exome array analysis identifies new loci and low-frequency variants influencing insulin processing and secretion. Nat Genet. 2013;45(2): 197-201.

32. Wu MC, Kraft P, Epstein MP, et al. Powerful SNP-set analysis for case-control genome-wide association studies. Am J Hum Genet. 2010;86(6):929-942.

33. Feng S, Liu D, Zhan X, Wing MK, Abecasis GR. RAREMETAL: fast and powerful meta-analysis for rare variants. Bioinformatics. 2014;30(19):2828-2829.

34. Purcell SM, Wray NR, Stone JL, et al.; International Schizophrenia Consortium. Common polygenic variation contributes to risk of schizophrenia and bipolar disorder. Nature. 2009;460(7256):748-752.

35. Campbell CD, Kirby A, Nemesh J, Daly MJ, Hirschhorn JN. A survey of allelic imbalance in F1 mice. Genome Res. 2008;18(4):555-563.

36. Wajnrajch M, Meng Y, Dauber A, et al. Supplemental Table 1. Figshare, 2020. Posted June 14, 2020. doi:10.6084/ m9.figshare.12479090.v1.

37. Lettre G, Butler JL, Ardlie KG, Hirschhorn JN. Common genetic variation in eight genes of the GH/IGF1 axis does not 
contribute to adult height variation. Hum Genet. 2007;122(2): 129-139.

38. D'Aloisio AA, Schroeder JC, North KE, et al. IGF-I and IGFBP-3 polymorphisms in relation to circulating levels among African American and Caucasian women. Cancer Epidemiol Biomarkers Prev. 2009;18(3):954-966.

39. Guo MH, Stoler J, Lui J, et al. Redefining the progeroid form of Ehlers-Danlos syndrome: report of the fourth patient with
B4GALT7 deficiency and review of the literature. Am J Med Genet A. 2013;161A(10):2519-2527.

40. Stevens A, De Leonibus C, Whatmore A, et al. Pharmacogenomics related to growth disorders. Horm Res Paediatr. 2013;80(6):477-490.

41. Ouni M, Belot MP, Castell AL, Fradin D, Bougnères P. The P2 promoter of the IGF1 gene is a major epigenetic locus for GH responsiveness. Pharmacogenomics J. 2016;16(1):102-106. 